A. Weisbecker, M. Burmester \& A. Schmidt (Hrsg.): Mensch und Computer 2015 Workshopband, Stuttgart: Oldenbourg Wissenschaftsverlag, 2015, S. 367-376.

\title{
Gamification User Types zur Unterstützung der Konzeption in der Softwareentwicklung
}

\author{
Sebastian Korbas \\ Centigrade GmbH, Niederlassung Mülheim a. d. Ruhr
}

\section{Zusammenfassung}

Dieser Beitrag stellt die Verwendung von Gamification User Types als unterstützendes Element im Rahmen des Designprozesses von Software-Applikationen vor. Anhand der Erhebung und Klassifizierung von fünf verschiedenen Nutzertypen mit spielerischem Hintergrund wurde von der Firma Centigrade $\mathrm{GmbH}$ in einem konkreten Anwendungsfall das motivationale Konzept für die User Experience einer Smartphone App gestaltet. Hierzu wurde eine explorative Studie mit 121 Probanden durchgeführt.

\section{$1 \quad$ Einleitung}

Im Zuge von nutzerzentrierten Designprozessen kommt es regelmäßig vor, dass der Konzeption in Abhängigkeit von der jeweiligen Projektlage oder den geltenden Rahmenbedingungen nur schwer ein konkreter Nutzer zu Grunde gelegt werden kann. Beispiele hierfür sind etwa Produktentwicklungen für die Forschung, Designstudien oder Projekte, die eine große und weit gestreute Nutzergruppe adressieren. Um an die relevanten Daten zur Skizzierung von Personas und ihren Bedürfnissen sowie Anforderungen zu gelangen, sind empirische Studien von Nöten, die Charaktereigenschaften, Persönlichkeitsmerkmale oder motivationale Beweggründe insbesondere in Bezug auf die Nutzerinteraktion mit dem System offenbaren. Im Bereich der psychologischen Forschungsmethodik steht dazu eine Vielzahl an Instrumenten zur Verfügung. Häufig weisen diese aber eine entweder zu hohe (zu spezifisch auf einen expliziten Anwendungsfall zugeschnittene) oder zu geringe (zu generalisierte Aspekte der Persönlichkeit erfassende) Spezialisierung auf, um in einem technikbezogenen Kontext mit motivationalen Komponenten valide Messergebnisse im Sinne des Designprozesses zu liefern. Im Bereich der Gamification können solche, an der motivationalen Basis des Nutzers erhobenen Daten jedoch entscheidend für die Annahme oder Ablehnung eines Systems sein. Falsch eingesetzte Motivatoren, die in einem Fall erfolgreich funktionieren, können in einem 
anderen, ähnlich gelagerten Kontext das Gegenteil bewirken. Die Beispiele hierfür sind mittlerweile zahlreich und es zeigte sich dabei ebenfalls, das häufig bereits kleine Details der motivationalen Ausrichtung einen starken Einfluss auf die gesamte User Experience haben können (vgl. Schering, Niesenhaus \& Schmidt, 2014). Es liegt also nahe, zu Beginn des Designprozesses von Gamification-Projekten zunächst eine Erhebung ebendieser Nutzermotivatoren durchzuführen.

Im Bereich der Videospieleforschung finden sich ähnliche Ansätze, um Spieler gemäß eines vorliegenden Musters anhand ihres Verhaltens im Spiel zu klassifizieren. Am häufigsten trifft man im Rahmen dessen auf die Spielertypen-Taxonomie von Richard Bartle (1996). Diese klassifiziert Spieler in vier Kategorien: Achiever, Explorer, Killer und Socializer. Was hierbei jedoch ebenso häufig außer Acht gelassen wird ist, dass Bartle über seine Taxonomie selbst aussagt, dass diese aufgrund ihrer qualitativen Erhebung zu spezifisch auf so genannte MUD-Spieler (Multi-User-Dungeon) zugeschnitten ist und somit zu exklusiv ist, um ohne weiteres auf einen anderen Kontext angewendet werden zu können (Bartle, 1996). Im Umfeld der jüngeren Fachliteratur zum Thema Gamification wurde bereits begonnen, auf diesen Bedarf zu reagieren, indem von den Autoren verschiedene Modelle von GamificationNutzertypen skizziert wurden. Diese verfolgen verschiedene Ansätze, wie etwa eine generelle Teilung in intrinsisch und extrinsisch motivierte Spieler (Marczewski, 2013a) oder die Entkopplung von einem allgemeinen Nutzertypen hin zu einem sich im Kontext der aktuell dargebotenen Inhalte veränderndem Typus (Canossa \& Drachen, 2009). Dazu variiert je nach Modell außerdem die Anzahl der Klassifikationen. Aktuelle Konzepte differenzieren hier momentan beispielsweise in acht Unterteilungen (vgl. Radoff, 2011; Herger, 2014). Allen Ansätzen gleich ist jedoch, dass diese bislang nicht ausreichend und in größerem Maßstab über den Konzeptionsstatus hinaus untersucht und empirisch evaluiert wurden. Die einzige groß angelegte Studie, die Gamification-Nutzertypen adressiert, wird derzeit von Andrzej Marczweski (2015) durchgeführt und befindet sich momentan in zweiter Iteration in der Datenerhebungsphase (Stand: 05/2015).

Der Mangel und somit gleichzeitige Bedarf nach verlässlichen Messinstrumenten zur Klassifikation von Nutzertypen mit motivationalen Hintergründen für die Designpraxis wird somit deutlich. Insbesondere, da diese als Grundlage im industriellen Gamification-Kontext fehlen und dadurch immer noch Systeme an den Endnutzer gelangen, deren motivationale Konzepte nicht ausreichend auf den Kontext abgestimmt sind. In der Folge empfiehlt es sich für Designer in diesem Bereich, je nach Projektumfang und Ressourcen selbst einen eigenen, spezifisch abgestimmten Fragebogen zu entwickeln und eine Evaluation damit durchzuführen.

\section{Marczewski’s intrinsisches Nutzertypenmodell}

Als theoretische Grundlage dieses Fragebogens sollte Marczewski's intrinsisches Nutzertypenmodell (2013b) dienen. Die Entscheidung hierzu fiel, da Marczewski bereits Erfahrungen in der Evaluation seiner Nutzertypen in erster Iteration mit 426 Probanden sammeln und seine Nutzertypen dahingehend anpassen konnte (Marczewski, 2013c). Hierbei ist er von einer vergleichbaren intrinsischen Basis wie Bartle (1996) ausgegangen, hat die hier zu 
Grunde liegenden intrinsischen Motive jedoch weiter ausgebaut und generalisiert. Außerdem hat er die vier bestehenden Nutzertypen um noch zwei weitere ergänzt. Abbildung 1 veranschaulicht dieses Modell.

Unter dem Philanthropist, also dem Menschenfreund, versteht man einen sozial eingestellten Nutzer, der gerne Verantwortung trägt und sich um andere kümmert. Er agiert bevorzugt für ein größeres Ziel, dem eine höhere Bedeutung oder ein tieferer Sinn beziehungsweise Nutzen innewohnt. Nutzertypen dieser Kategorie sind häufig Tutoren oder Betreuer und in sozialen Projekten oder Vereinen engagiert. Ebenfalls auf eine soziale Basis baut auch der Socialiser. Dieser Typus ist aber mehr an der Interaktion mit anderen interessiert. Für ihn stehen die Kommunikation und der Vergleich mit anderen im Vordergrund. Eher auf eigene Vorteile bedacht ist hingegen der Player. Er wird am stärksten von „klassischen“ GamificationMechanismen angetrieben wie etwa dem Willen, der Beste in einer Tätigkeit zu sein und stets auf den vordersten Plätzen zu rangieren. Das Gewinnen aber auch das Erleben einer Aktion um ihrer selbst Willen machen für diesen Typus einen großen Anreiz aus.

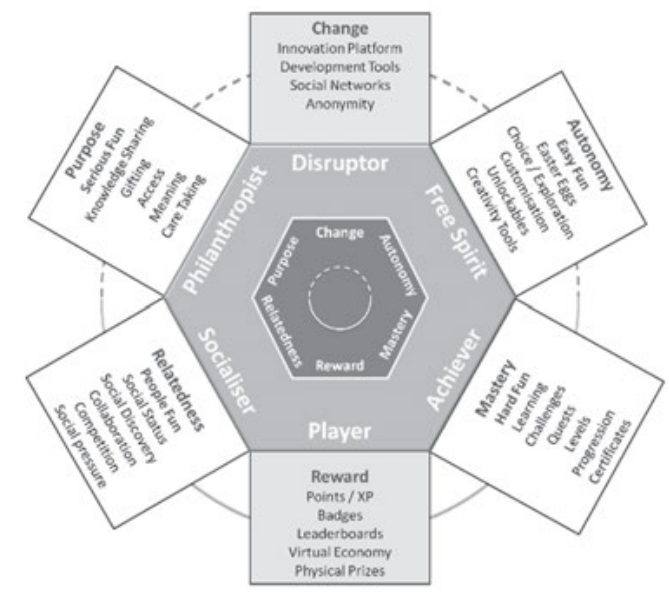

Abbildung 1: Illustration des intrinsischen NutzertypenModells von Marczewski (2013b). (C) Gamified UK 2014
Es gibt unter vielen Anwendern - vor allem im Spielebereich - auch einen großen Anteil, der seine Erfüllung in kreativer Freiheit und dem Bestreiten neuer, unkonventioneller Wege sucht. Diese werden von Marczewski als Free Spirits, also Freigeister, bezeichnet.

Ein weiterer für die Fragebogenkonstruktion verwendeter Nutzertyp ist der Achiever. Dieser kann frei als „Sammler“ übersetzt werden. Achiever sind am zufriedensten, wenn sie bei ihren Handlungen einen Fortschritt wahrnehmen und klar kommunizierte Ziele erreichen können. Sie sind häufig entwicklungsgetrieben und wollen Sachverhalte in ihrer Komplexität erfassen und darin eintauchen.

Die Nutzertypen des Socialisers und Achievers entsprechen in ihren Grundzügen ihren Gegenstücken in Bartle's Modell. Artverwandt mit Bartle's Explorer ist Marczewski's Free Spirit. Das Modell des Killers wurde von Marczewski hingegen entschärft und in den Player umgewandelt. Neben dem oben bereits erwähnten Philanthropist führt Marczewski noch einen weiteren Nutzertypen an, der sich nicht bei Bartle findet: den Disruptor. Dieser Nutzer wird dadurch angetrieben, dass er bestehende Systeme kompromittieren und Schlupflöcher aufdecken will. Er ist als eine Art negierter Explorer zu verstehen. Allerdings wurde dieser Nutzertyp aufgrund seiner Charakteristik nicht im Rahmen des hier vorgestellten Fragebogens berücksichtigt, da der explorative Fokus der Erhebung lediglich projektrelevante Nutzertypen zum Ziel haben sollte. 


\section{Fragebogenkonstruktion}

Der hier vorgestellte Fragebogen wurde im Rahmen einer Designstudie entwickelt. Diese hatte zum Ziel, eine Smartphone App im Kontext der Energiewende zu konzipieren, die ihre Anwender durch gamifizierte Elemente in einem nachhaltigeren Lebensstil unterstützen sollte.

\subsection{Pretest}

Im ersten Schritt der Fragebogenkonstruktion wurden zu jedem der oben beschriebenen fünf Nutzertypen jeweils vier Aussagen generiert. Diese wurden in einer vorangestellten Erhebung qualitativ auf ihre Aussagekraft hin überprüft. Die Stichprobe des Pretests bestand dazu aus je vier weiblichen und männlichen Probanden, die zunächst kurze Zusammenfassungen der Spielertypen vorgelegt bekamen und anschließend die generierten Aussagen diesen zuordnen sollten. Anhand der Erkenntnisse dieser Erhebung wurden die Aussagen angepasst und um vier weitere Items ergänzt (siehe: Tabelle 1).

\begin{tabular}{|c|c|}
\hline $\mathrm{Nr}$. & Aussage (Item) \\
\hline 1 & Mir gefällt es, wenn ich einen steten Fortschritt bei den Dingen erkennen kann, die ich tue. \\
\hline 2 & Ich bin kreativ und gestalte oder entwerfe gerne Dinge. \\
\hline 3 & Ich helfe gerne anderen. \\
\hline 4 & Ich tausche mich gerne online mit anderen aus. \\
\hline 5 & Ich teile mein Wissen gern mit anderen. \\
\hline 6 & Ich bin wissensdurstig und bilde mich gerne weiter. \\
\hline 7 & Für mich sind Dinge wichtig, die einen tieferen Sinn oder Nutzen für das Allgemeinwohl haben. \\
\hline 8 & Ich mache anderen gerne Geschenke oder bereite ihnen kleine Aufmerksamkeiten. \\
\hline 9 & Es motiviert mich, auf eine bestimmte Sache oder Option hinzuarbeiten bzw. darauf zu sparen. \\
\hline 10 & Ich verbringe oft viel Zeit mit dem Ausprobieren einer Sache. \\
\hline 11 & Auf persönliche Erfolge bin ich stolz und zeige diese gerne. \\
\hline 12 & Mir gefällt es, gemeinsam mit anderen etwas zu erreichen. \\
\hline 13 & Ich versuche oft, der Beste in dem zu sein, was ich tue. \\
\hline 14 & Ich arbeite Dinge gerne in festen Etappen ab. \\
\hline 15 & $\begin{array}{l}\text { Ich beteilige mich gerne an Dingen, wenn dadurch anderen direkt und sichtbar geholfen wird (bspw. } \\
\text { Spendenaktionen für Menschen in Not, Tierhilfe, Umweltschutz, etc.). }\end{array}$ \\
\hline 16 & $\begin{array}{l}\text { Ich wäge meine Handlungen objektiv ab, damit ich immer weiß, wie viel ich leiste und was ich zu } \\
\text { bekommen habe. }\end{array}$ \\
\hline 17 & $\begin{array}{l}\text { Es bereitet mir besondere Freude, wenn ich bei einer Sache versteckte Hinweise oder Anspielungen } \\
\text { entdecken kann. }\end{array}$ \\
\hline 18 & Ich wähle gerne aus vielen Möglichkeiten aus. \\
\hline 19 & Ich messe mich gerne im Wettbewerb oder Vergleich. \\
\hline 20 & $\begin{array}{l}\text { Wenn ich mich an etwas beteilige, stehen dabei für mich in erster Linie Ergebnisse und Vergütungen im } \\
\text { Vordergrund. }\end{array}$ \\
\hline 21 & Ich achte stets darauf, vor anderen gut dazustehen. \\
\hline 22 & Ich bin umso motivierter, wenn andere mir bei einer Sache zusehen. \\
\hline 23 & Es macht mich besonders stolz, wenn ich für eine Leistung eine Urkunde oder Auszeichnung bekomme. \\
\hline 24 & Ich habe Freude am sammeln bestimmter Objekte (bspw.: Schallplatten, Comics, Briefmarken, etc.). \\
\hline
\end{tabular}

Tabelle 1: Generierte Items des Gamification User Type Tests nach der Pretest-Anpassung. 


\subsection{Fragebogen (Onlinestudie)}

Der finale Fragebogen zur Nutzertypenerfassung wurde als Onlinestudie konzipiert und sollte möglichst schnell durchführbar und schlank aufgebaut sein. Dieser Umstand ist maßgeblich auf die Projektplanung mit dementsprechend eng kalkulierten Zeitressourcen und dem ihr zu Grunde gelegten Lean UX-Designprozess zurückzuführen. Auf explorativer Ebene soll schnell zu verwertbaren Ergebnissen gelangt werden, an denen sich iterativ der Designprozess aufbauen kann. So wurden zu Beginn der Befragung zur Skizzierung von Personas die demografischen Daten Geschlecht, Alter, Beruf und Bildungsstand abgefragt. Anschließend folgten vier Fragen zum Lebensstil der Probanden. Dazu gehörte die Größe des Haushalts in Personen, ob der Proband diesen selbst führt, ob er ein Smartphone oder Tablet benutzt und wie er sich selbst im Hinblick auf seine Nachhaltigkeit im Alltag einschätzt (4stufige Likert-Skala). Hieran setzte nun der Kern der Umfrage an: Vier nacheinander geschaltete Blöcke mit je sechs Items, aus denen der Proband via Checkboxes maximal zwei auswählen konnte, mit denen er sich am meisten identifizierte. Alternativ konnte er auch kein Feld ankreuzen. In jedem dieser Item-Blöcke adressierte jeweils ein Item konkret einen einzelnen der fünf Nutzertypen. Zusätzlich war aber auch noch je ein hybrides Item unter der Auswahl, das auf zwei Nutzertypen verwies. Somit fokussierte der Fragebogen eine schnelle und im Entwicklerteam interdisziplinär auswertbare Ergebnisform auf deskriptiver Ebene. Komplexe inferenzstatistische Verfahren standen nicht im Mittelpunkt der Erhebung und bildeten nur einen Randaspekt der Studie aus.

\section{Ergebnisse}

\subsection{Stichprobe}

An der Online-Studie nahmen 121 Probanden in einem Zeitraum von 12 Tagen teil. Davon waren 57 (47.1\%) Teilnehmer männlich und 64 (52.9\%) weiblich. Die Teilnehmer waren zwischen 16 und 63 Jahren alt (M=31.40, SD=9.924). 116 (95.9\%) der Teilnehmer gaben an, ein Smartphone oder Tablet zu benutzen. Von den befragten Versuchsteilnehmern verfügten 46 (38.0\%) über ein abgeschlossenes Studium, 42 (34.7\%) über das Abitur/Fachabitur, 23 (19.0\%) über den Realschulabschluss beziehungsweise die mittlere Reife und 6 (5.0\%) über den Hauptschulabschluss. Eine Person (0.8\%) gab an, keinen Abschluss zu besitzen. Drei Personen (2.5\%) nannten „Sonstiges“ als ihren Bildungsstand. 23 (19.0\%) der Probanden gaben an, alleine zu leben. 46 (38.0\%) der Probanden lebten zum Zeitpunkt der Studie zu zweit. 24 (19.8\%) der Versuchspersonen wohnten mit insgesamt drei, 22 (18.2\%) mit insgesamt vier, fünf $(4.1 \%)$ mit insgesamt fünf und ein Teilnehmer $(0.8 \%)$ mit insgesamt sechs Personen in einem Haushalt. Davon gaben 99 (81.8\%) der Versuchsteilnehmer an, ihren Haushalt selbst zu führen. Die Frage, ob sich die Probanden für Nachhaltigkeit interessieren (vierstufige Likert-Skala von ,kaum“ $=0$ bis , ,ja, sehr" $=3$ ) beantworteten 7 Probanden $(5.7 \%)$ mit „,kaum“, 32 Probanden (26.4\%) mit ,ein wenig“, 64 (52.9\%) mit ,ja“ und 18 Probanden (14.9\%) mit ,ja, sehr“ (M=1.77; SD=0.772). 
Die Berufsbilder der Versuchspersonen waren sehr vielseitig. Dies stellt insofern einen positiven Aspekt dar, dass die Energiewende mit ihrer komplexen Thematik ebenfalls quer durch alle Gesellschaftsschichten und Berufsbilder die verschiedensten Menschen betrifft. Bespiele aus der Stichprobe sind etwa Ärzte, Bestatter, Flugbegleiter, Hausfrauen, kaufmännische Angestellte, Raumpfleger, Softwareentwickler und Wirtschaftsingenieure.

\subsection{Inferenzstatistische Aspekte}

Als Ergänzung zu den deskriptiven Daten, die den Schwerpunkt des Fragebogens bilden, wurden die erhobenen Daten zusätzlich im Hinblick auf mögliche Zusammenhänge hin untersucht. Ein durchgeführter Mann-Whitney-U-Test (UV=Geschlecht, AV=Nachhaltigkeit) in Bezug auf die angegebene Nachhaltigkeit von Frauen und Männern ergab hier einen signifikanten Unterschied zwischen den Geschlechtern ( $U=1388.5, Z=-2.482, \mathrm{p}=.013, \mathrm{r}=-.226$ ). Dabei hatten Frauen einen Mittelwert von $\mathrm{M}=1.91 \quad(\mathrm{SD}=0.811)$ und Männer einen von $\mathrm{M}=1.61$ ( $\mathrm{SD}=0.701$ ). Zur Untersuchung der Nachhaltigkeit von über und unter 30-jährigen Probanden wurde die Stichprobe in zwei Gruppen geteilt. Gruppe 1 der unter 30-Jährigen bestand aus 69 Probanden und hatte einen Nachhaltigkeitswert von $\mathrm{M}=1.55$ ( $\mathrm{SD}=0.796$ ). Gruppe 2 der über 30-Jährigen bestand hingegen aus 52 Probanden mit einem Mittelwert der Nachhaltigkeit von $\mathrm{M}=2.06$ ( $\mathrm{SD}=0.639$ ). Der Vergleich der Mittelwerte durch die Berechnung eines weiteren Mann-Whitney-U-Tests (UV=Alter, AV=Nachhaltigkeit) ergab hier einen signifikanten Unterschied der Gruppen bezüglich der Nachhaltigkeit ( $U=1188, \mathrm{Z}=-$ 3.483, $\mathrm{p}=<.001, \mathrm{r}=-.317$ ). Über 30-jährige Probanden schätzen sich als signifikant nachhaltiger im Alltag ein. Ein durchgeführter Kruskal-Wallis-Test zeigte hingegen keinen signifikanten Unterschied zwischen der Haushaltsgröße der Probanden und ihrer angegebenen Nachhaltigkeit.

\subsection{Deskriptive Aspekte}

Die von den Probanden selektierten Aussagen wurden, den Nutzertypen entsprechend, farblich codiert und in eine Blockansicht übertragen. Hat ein Proband nur eine Aussage in einem Fragenblock ausgewählt, so wurde diese doppelt gewertet. Hieraus ergibt sich für jeden Probanden ein farbliches Muster aus acht Blöcken. Diese wurden anschließend nach ihrer Häufigkeit absteigend geordnet. Die Betrachtung der geordneten Ergebnisse erfolgte nun in zwei Abstufungen:

- Welche Nutzertypen kommen bei jedem Probanden absolut am häufigsten vor (1. Grad)?

- Welche Nutzertypen kommen bei jedem Probanden absolut am zweithäufigsten vor (2. Grad)?

Die Betrachtung in zwei Graden dient hierbei dem Zweck, einen generellen Fokus für das Gamification Design ausrichten zu können (1. Grad), aber über dies hinaus noch eine weitere Richtung für einen Gestaltungsraum (vgl. bspw. Schering 2014) von Nebenaspekten, sekundären Motivatoren oder konzeptionellen Erweiterungen definieren zu können (2. Grad). 
Das folgende Beispiel (Abbildung 2) soll diese Aufschlüsselung verdeutlichen:

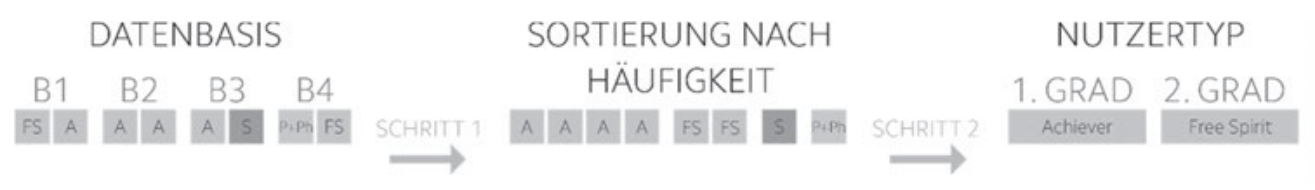

Abbildung 2: Veranschaulichung der Ableitung von Nutzertypen 1. und 2. Grades. Typ-Legende: A=Achiever, FS=Free Spirit, $P h=$ Philanthropist, $S=$ Socialiser, $P=$ Player .

Anhand der Farbtabelle (Abbildung 3) lässt sich in der Praxis schnell erkennen, dass beispielsweise dieser Proband hauptsächlich den Achievern (orange) und in weiterer Betrachtung den Free Spirits (grün) zuzuordnen ist.

S SOCIALIZER

FS FREE SPIRIT (EXPLORER)

A ACHIEVER

P PLAYER (KILLER)
Ph PHILANTHROPIST

P+S DUO: PLAYER (KILLER)+SOCIALIZER

A+FS DUO: ACHIEVER+FREE SPIRIT (EXPLORER)

P+Ph DUO: PLAYER (KILLER)+PHILANTHROPIST

Abbildung 3: Farbtabelle zur Codierung der Nutzertypen. Typ-Legende: A=Achiever, FS=Free Spirit, Ph=Philanthropist, $S=$ Socialiser, $P=$ Player.

Nach der Auswertung des gesamten Datensatzes ergaben sich dann folgende Verteilungen der Nutzertypen (Abbildung 4 \& 5):

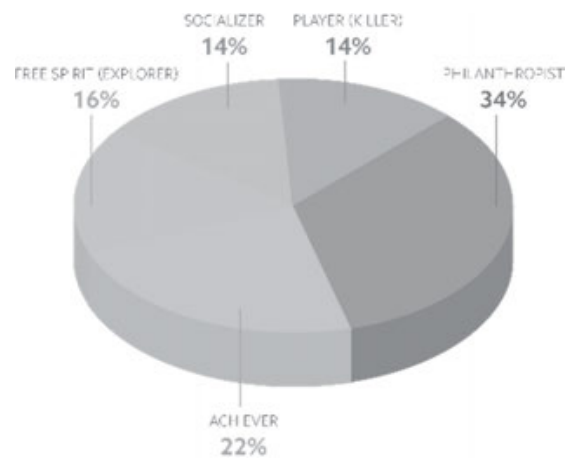

Abbildung 4: Erhobene Nutzertypen 1. Grades.

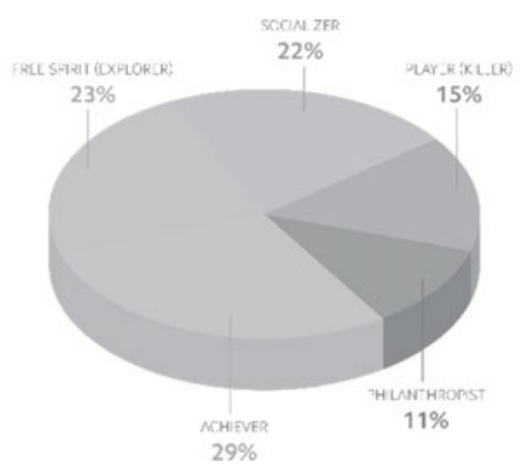

Abbildung 5: Erhobene Nutzertypen 2. Grades.

Die erhobenen Aussagen (Items) wurden in ihren absoluten und relativen Häufigkeiten wie folgt erfasst (siehe: Tabelle 2). 


\begin{tabular}{|c|c|c|c|c|c|}
\hline Nr. & Typ & Aussage (Item) & Motiv & \multicolumn{2}{|c|}{$\begin{array}{l}\text { Abs.+Rel. } \\
\text { Häufigkeit }\end{array}$} \\
\hline 1 & A & $\begin{array}{l}\text { Mir gefällt es, wenn ich einen steten Fortschritt bei den Dingen erken- } \\
\text { nen kann, die ich tue. }\end{array}$ & Progression & 87 & $71,9 \%$ \\
\hline 2 & FS & Ich bin kreativ und gestalte oder entwerfe gerne Dinge. & $\begin{array}{l}\text { Customization / } \\
\text { Creativity Tools }\end{array}$ & 60 & $49,9 \%$ \\
\hline 3 & $\mathrm{Ph}$ & Ich helfe gerne anderen. & Care Taking & 59 & $48,8 \%$ \\
\hline 4 & S & Ich tausche mich gerne online mit anderen aus. & Social Discovery & 56 & $46,3 \%$ \\
\hline 5 & $\mathrm{Ph}$ & Ich teile mein Wissen gern mit anderen. & Knowledge Sharing & 56 & $46,3 \%$ \\
\hline 6 & A & Ich bin wissensdurstig und bilde mich gerne weiter. & Learning & 52 & $43,0 \%$ \\
\hline 7 & $\mathrm{Ph}$ & $\begin{array}{l}\text { Für mich sind Dinge wichtig, die einen tieferen Sinn oder Nutzen für } \\
\text { das Allgemeinwohl haben. }\end{array}$ & Meaning & 49 & $40,5 \%$ \\
\hline 8 & $\mathrm{Ph}$ & $\begin{array}{l}\text { Ich mache anderen gerne Geschenke oder bereite ihnen kleine Aufmerk- } \\
\text { samkeiten. }\end{array}$ & Gifting & 48 & $39,7 \%$ \\
\hline 9 & $\mathrm{~A}+\mathrm{FS}$ & $\begin{array}{l}\text { Es motiviert mich, auf eine bestimmte Sache oder Option hinzuarbeiten } \\
\text { bzw. darauf zu sparen. }\end{array}$ & Unlockables & 48 & $39,7 \%$ \\
\hline 10 & FS & Ich verbringe oft viel Zeit mit dem Ausprobieren einer Sache. & Exploration & 42 & $34,7 \%$ \\
\hline 11 & $\mathrm{P}+\mathrm{S}$ & Auf persönliche Erfolge bin ich stolz und zeige diese gerne. & Social Status & 40 & $33,1 \%$ \\
\hline 12 & S & Mir gefällt es, gemeinsam mit anderen etwas zu erreichen. & Collaboration & 39 & $40,5 \%$ \\
\hline 13 & $\mathrm{P}$ & Ich versuche oft, der Beste in dem zu sein, was ich tue. & Leaderboards & 32 & $26,4 \%$ \\
\hline 14 & A & Ich arbeite Dinge gerne in festen Etappen ab. & Levels & 28 & $23,1 \%$ \\
\hline 15 & $\mathrm{P}+\mathrm{Ph}$ & $\begin{array}{l}\text { Ich beteilige mich gerne an Dingen, wenn dadurch anderen direkt und } \\
\text { sichtbar geholfen wird (bspw. Spendenaktionen für Menschen in Not, } \\
\text { Tierhilfe, Umweltschutz, etc.). }\end{array}$ & $\begin{array}{l}\text { Physical Prizes / } \\
\text { Meaning / Care } \\
\text { Taking }\end{array}$ & 26 & $21,5 \%$ \\
\hline 16 & $P$ & $\begin{array}{l}\text { Ich wäge meine Handlungen objektiv ab, damit ich immer weiß, wie } \\
\text { viel ich leiste und was ich zu bekommen habe. }\end{array}$ & (Virtual) Economy & 24 & $19,8 \%$ \\
\hline 17 & FS & $\begin{array}{l}\text { Es bereitet mir besondere Freude, wenn ich bei einer Sache versteckte } \\
\text { Hinweise oder Anspielungen entdecken kann. }\end{array}$ & Easter Eggs & 23 & $19,0 \%$ \\
\hline 18 & FS & Ich wähle gerne aus vielen Möglichkeiten aus. & Choice & 22 & $18,2 \%$ \\
\hline 19 & $\mathrm{P}+\mathrm{S}$ & Ich messe mich gerne im Wettbewerb oder Vergleich. & Competition & 16 & $13,2 \%$ \\
\hline 20 & $\mathrm{P}$ & $\begin{array}{l}\text { Wenn ich mich an etwas beteilige, stehen dabei für mich in erster Linie } \\
\text { Ergebnisse und Vergütungen im Vordergrund. }\end{array}$ & $\begin{array}{l}\text { Rewards / Physical } \\
\text { Prizes }\end{array}$ & 13 & $10,7 \%$ \\
\hline 21 & S & Ich achte stets darauf, vor anderen gut dazustehen. & $\begin{array}{l}\text { Social Status / Social } \\
\text { Pressure (neg.) }\end{array}$ & 13 & $10,7 \%$ \\
\hline 22 & S & Ich bin umso motivierter, wenn andere mir bei einer Sache zusehen. & Social Pressure (pos.) & 11 & $9,1 \%$ \\
\hline 23 & $P$ & $\begin{array}{l}\text { Es macht mich besonders stolz, wenn ich für eine Leistung eine Urkun- } \\
\text { de oder Auszeichnung bekomme. }\end{array}$ & Badge / Virtual Prizes & 11 & $9,1 \%$ \\
\hline 24 & A & $\begin{array}{l}\text { Ich habe Freude am sammeln bestimmter Objekte (bspw.: Schallplatten, } \\
\text { Comics, Briefmarken, etc.). }\end{array}$ & Completion & 10 & $8,3 \%$ \\
\hline
\end{tabular}

Tabelle 2: Sortierung der Item-Selektionen nach Häufigkeiten. Typ-Legende: A=Achiever, FS=Free Spirit, Ph=Philanthropist, $S=$ Socialiser, $P=$ Player. Die absoluten Häufigkeiten zeigen die tatsächlichen Nutzerselektionen an. Die relativen Häufigkeiten zeigen an, wie vie 


\section{Diskussion \& Ausblick}

Im Falle dieser Designstudie half die Erhebung der Probandendaten dem Designprozess zwei stereotype Nutzermodelle - den Philanthropist und den Achiever - zu Grunde zu legen, an denen sich die Konzeption orientieren kann. Entscheidend wirkt sich dies besonders bei der Wahl der zu verwendenden Gamification-Elemente aus. Hier gibt es bekanntlich Mechaniken, die bei falscher Kombination oder unüberlegtem Einsatz ein System nicht motivational anreichern, sondern für den falschen Nutzertypen gar eine konträre, demotivierende Wirkung haben können. Abbildung 6 zeigt den aus den Daten generierten Gestaltungsraum für Gamification Mechaniken im ersten und zweiten Grad an.

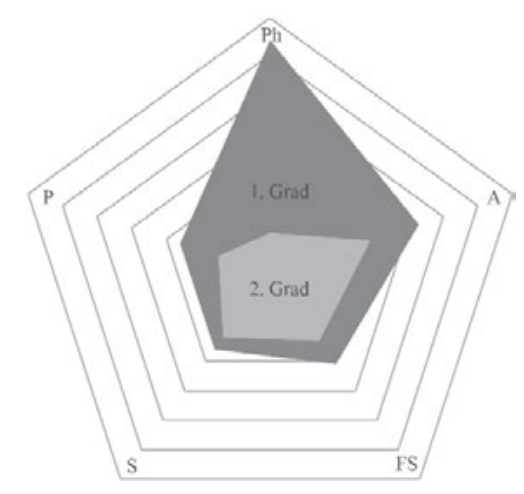

Abbildung 6: Gestaltungsraum für den Designprozess der Gamification Mechaniken. Typ-Legende: A=Achiever, FS=Free Spirit, Ph=Philanthropist, $S=$ Socialiser, $P=$ Player .
Bezieht man die Motive hinter den einzelnen Nutzertypen (vgl. Tabelle 2) mit ein, ergibt sich eine Struktur, die das motivationale Rückgrat der Applikationsinteraktion bilden sollte. Elemente dieser sind im vorliegenden Falle beispielsweise Care Taking, Social Discovery, Knowledge Sharing und Gifting, die in einen klar kommunizierten Rahmen (Progression) gefasst werden müssten, der wiederum einen gewissen Raum für individuelle Gestaltung (Customization) zulässt. Es wird somit deutlich, dass die Bestimmung der Nutzertypen zwar die Richtung des Designs auf übergeordneter Ebene vorgibt, die Feinheiten und Details aber durchaus auch von anderen Nutzertypen stammen können, um ein in Bezug zur Nutzergruppe rundes Gamification Konzept abzuleiten.

Die Unterteilung des vorliegenden Fragebogens in die kontextabhängigen demografischen Daten und den hinsichtlich allgemeiner Aussagekraft generierten Gamification-Nutzertypen erlaubt generell die Übertragung auf andere Anwendungsfälle. Als ausbaufähige Erweiterungen des Fragebogenkonzepts bezüglich der Vereinfachung und Optimierung von Gamification Designprozessen bieten sich künftig aber noch zwei weitere Optionen an: Zum einen wäre hier die Entwicklung eines Messinstruments zur Evaluation der motivationalen Wirkung von Gamification-Konzepten und Prototypen nach der ersten Designiteration bis hin zum finalen Produkt zu nennen; zum anderen eine Taxonomie der Gamification Mechaniken und ihrer Motive anhand des Gestaltungsraumes aus Abbildung 6, mittels der sich die jeweils zu den Nutzertypen passenden Mechaniken expliziter bestimmen ließen.

Insgesamt stellte die Durchführung des hier vorgestellten Fragebogens im Rahmen des Gamification Designs jedoch bereits in dieser Form eine große Bereicherung hinsichtlich der Erfassung der motivationalen Ansprüche der potentiellen Nutzer dar. In der Regel bieten 
Projektarbeiten und kundenseitiges Management häufig wenige Möglichkeiten, eine komplexe und schwer beobachtbare Größe wie die Motivation angemessen erfassen zu können. Besonders hier lieferte die Studie bei relativ geringem Ressourcenaufwand wichtige Anhaltspunkte auf die nachfolgende Designentscheidungen gestützt werden konnten.

\section{Literaturverzeichnis}

Bartle, R. (1996). Hearts, clubs, diamonds, spades: Player who suit MUDs. Abgerufen am 11. Oktober 2014 von http://www.mud.co.uk/richard/hcds.htm

Canossa, A., \& Drachen, A. (2009). Patterns of Play: Play-Personas in User-Centered Game Development. Breaking New Ground: Innovation in Games, Play, Practice and Theory. Proceedings of DiGRA 2009. London: DiGRA.

Herger, M. (2014). Enterprise Gamification. Marston Gate: Amazon.co.uk, Ltd.

Marczewski, A. (2013a) Extrinsically and Intrinsically Motivated User Types. Abgerufen am 13. Oktober 2014 von Gamified UK: http://www.gamified.co.uk/2013/07/08/extrinsic-andintrinsically-motivated-user-types/\#.VDroIqKIx7p

Marczewski, A. (2013b) User Types 2.0 - The Hexad. Abgerufen am 12. Oktober 2014 von Gamified UK: http://www.gamified.co.uk/user-types/\#.VDrdW6KIx7q

Marczewski, A. (2013c) Gamification User Type Test - User Types 1.0 Test Results. Abgerufen am 12. Oktober 2014 von Gamified UK: http://www.gamified.uk/UserTypeTest/user-type-test-resultsold.php\#.VYvPzEaaKVw

Marczewski, A. (2015) Marczewski's User Type Test. Abgerufen am 01. Juni 2015 von Gamified UK: http://www.gamified.uk/UserTypeTest/user-type-test.php\#.VWxe90aaKVx

Radoff, J. (2011). Game On: Energize Your Business with Social Media. Indianapolis: Wiley Publishing, Inc.

Schering, S. (2014). Tagungsband des Gamification Workshops „Press Play?“. Ein Design Space für internale Gamification Anwendungen. Oldenbourg: De Gruyter.

Schering, S., Niesenhaus. J., Schmidt, R. (2014). Tagungsband des Gamification Workshops „Press Play? “. Oldenbourg: De Gruyter.

\section{Kontaktinformationen}

Sebastian Korbas

Centigrade $\mathrm{GmbH}$

Kreuzstr. 1-3, 45468 Mülheim a. d. Ruhr, Deutschland

sebastian.korbas@centigrade.de

www.centigrade.de 